\title{
A Method for Monitoring and Controlling Reproducibility of Intensity Data in Complex Electrospray Mass Spectra: A Thermometer Ion-based Strategy
}

\author{
Paolo Lecchi, Jinghua Zhao, Wesley S. Wiggins, Tzong-Hao Chen, \\ Ping F. Yip, Brian C. Mansfield, and John M. Peltier \\ Correlogic Systems, Inc., Rockville, Maryland, USA
} Reproducibility in mass spectral data is important in both biomarker discovery and spectral
database searching. We report a strategy, employing a series of substituted benzylpyridinium
thermometer ions that can be used to monitor changes in performance of multiple aspects of
an electrospray ionization source that impact the intensity axis of a spectrum. Performance
attributes, which could confound even isotope-based quantification strategies, are readily
assessed using a mixture of thermometer ions. Based on the observed behavior of the ions, a
procedure is proposed for monitoring instrument performance and compensating for factors
that affect reproducibility across both time and instruments. (J Am Soc Mass Spectrom 2009, 20,
$398-410$ ) () 2009 Published by Elsevier Inc. on behalf of American Society for Mass Spectrometry

$\mathrm{M}$ ass spectrometry (MS) has evolved into a technology that is much more widely adopted in fields beyond analytical chemistry than in previous decades. A significant reason for this acceptance is the improvement in both the reliability and performance of the instruments. In spite of these improvements, experimentalists are constantly pushing the envelope of instrumental capabilities to address new applications in chemistry, biochemistry, biology, medicine, and the interface between these fields.

One area of continuing challenge is to improve the reproducibility, especially in the intensity axis, of mass spectral data, to allow the comparison of new data to reference libraries or samples. For some forms of ionization, such as electron ionization (EI), reproducibility is sufficiently well controlled that spectra generated from different instrument types and/or from different mass spectrometer manufacturers [1], can be matched successfully to spectra in reference libraries. Other forms of ionization, such as electrospray ionization (ESI) and related atmospheric pressure ionization (API) techniques are less well controlled, and intensity reproducibility remains a challenge when trying to interpret new data by comparison to reference data. In the case of biomarker studies, researchers have tried to address the issue of reproducibility by use of isotope labeling [2, 3] or isotope dilution techniques [4], but problems remain [5]. Moreover, the extra layer of complexity that these methods create may increase the variability of the

Address reprint requests to Dr. J. M. Peltier, Correlogic Systems, Inc., 1405 Research Boulevard, Ste. 220, Rockville, MD 20850, USA. E-mail: jpeltier@correlogic.com analysis. Some of the commercial reagents also contribute a substantial cost to large-scale studies. As a result, label-free techniques $[6,7]$ are gaining in popularity, but these techniques still face a requirement for reproducibility across experiments and analytical platforms. Regardless of the approached used to discover them, once biomarkers are validated for a diagnostic application, their use in MS-based assays to classify clinical samples may be viewed as a specialized case of spectral library searching; albeit with a small library. In both applications, MS $m / z$ and intensity data are matched to some set of reference values. Therefore the analytical challenges with respect to reproducibility are the same.

Mass spectrometers employing atmospheric pressure ionization (API) techniques generally have yet to achieve the level of reproducibility of EI-MS systems [8-12]. Part of their variability may be attributed to the ionization process itself [13-16], because of competitive ionization and other suppression effects. However, even for simple samples analyzed in a mode in which potential differential ionization effects are negligible, differences in spectra between experiments and between instruments are still observed [8-10]. Some progress has been made [17-20] using various implementations of the calibration point concept [21, 22]. However, comparisons across instruments of the same class from different vendors, let alone different classes of instrument, in which the experimental time frame is different, remain challenging, especially when trying to reproduce ratios of peak abundances, rather than just looking at the presence or absence of peaks. Most recently, Hopley et al. [17] have demonstrated some ability to match spectra from multiple instruments to a
(C) 2009 Published by Elsevier Inc. on behalf of American Society for Mass Spectrometry. $1044-0305 / 09 / \$ 32.00$

doi:10.1016/j.jasms.2008.10.021
Published online November 6, 2008 Received August 19, 2008 Revised October 10, 2008 Accepted October 29, 2008 
small library of 48 compounds, yet they still experienced difficulties across some types of instruments. However, this work concentrated on MS/MS work in which a limited number of product ions are generated. This contrasts with the demands of biomarker studies in which many more ions must be sifted through, across a much broader $m / z$ range. Analysis and control of the reproducibility of such complex spectra is an ongoing area of research [23].

In considering the factors that might influence the observed ratios of detected ions in an API MS system, it is immediately apparent that the ions are produced in a much different environment than that in which they are detected. Thus, ions generated at atmospheric pressure must transit an atmosphere-vacuum interface that reduces the pressure by a factor of $10^{6}$ to $10^{10}$ depending on the instrument. This process is not completely reproducible for all ions at all $\mathrm{m} / \mathrm{z}$ ratios. During this transit, air and residual solvent vapors must be selectively removed, while transmitting enough of the charged analyte species to yield an analytically useful signal. This reduction in pressure is typically achieved in stages in which the ions pass successively through multiple ion optical elements, some of which consist of narrow apertures, separating one pressure zone from another. The number, orientation, and specific design of these elements differ by instrument type and manufacturer. These design differences complicate a uniform understanding of the factors affecting ion transmission. However, even with a given type of instrument, from a given manufacturer, where the design of the ion optics is fixed, there are transmission differences due to slight manufacturing differences in the relative dimensions and position of the optical components. Thus, there are both design-dependent and manufacturing-dependent effects that influence the intrinsic ion transmission characteristics of an API-MS system.

In addition to the inherent differences in ion transmission between instruments, there are time-dependent factors that affect ion transmission in a single instrument. Analyzed material is sprayed directly into the spectrometer leading, over time, to the coating, shortcircuiting, and possible corrosion of sensitive components. In addition, nonconductive materials deposited on the ion optics can acquire a charge and create so-called fringing fields, which modify the electric fields that transmit the ions and thereby affect ion transmission. Furthermore, the deposition of these contaminants can occlude the narrow apertures through which ions are transmitted. These occlusions can block the ion path or change the gas flows, from one pumping stage in the interface to another, thereby affecting ion transmission.

To improve reproducibility of API-based MS instruments, a better understanding of the impact of various instrumental and sample-related factors on the spectra is required. One approach to this is to find reporter ions that can measure changes within the different regions of the instrument and use them to independently detect changes in the generation, transmission, and detection of ions. Benzylpyridinium thermometer ions [24] are one such group of molecules. These molecules were originally applied to characterize the energy deposition in ions produced by secondary ion bombardment [25], and later used to characterize the internal energy deposition in ions traversing the first stage of pressure reduction in an electrospray ionization source. The ions have since been used to characterize energy deposition during the ionization process itself, in ESI, and other ionization modes [26-30].

Within the first stage of pressure reduction of an API source, there is a supersonic free expansion of atmospheric gases and the entrained analyte ions. During this process, analyte molecules undergo collisional energy transfers that may result in fragmentation (i.e., in-source collision-induced dissociation; CID). Many factors can influence this process [28], including the position of the secondary sampling orifice relative to the supersonic plume [28], temperature effects [31-34], the pressure and nature of the gas with which the analyte ions collide [24, 34], the nature of the solvent from which the ions are desorbed [8, 24], and the time scale between the activation of the ions in the source and their ultimate analysis [28]. Moreover, changes related to changes in local pressure and spray droplet evaporation, which affect ion temperatures, along with the distance between the sprayer tip and the sampling orifice have also been implicated $[29,35]$. Many of these studies have focused on the impact of the early stages of API sources on MS spectral content, or the impact of collision energy on MS/MS spectra. However, other stages of ion transmission, mass analysis, and ion detection, can have equally profound effects on spectral reproducibility.

Benzylpyridinium ions are useful probes of the dependency of energy deposition effects on API source conditions, in part because they have an intrinsic charge, making them less sensitive to variations in electrospray conditions than non-charged species. They are also chemically stable and exhibit good solubility in a wide range of solvent systems. By using benzylpyridinium ions with different substitutions, the energy of fragmentation can be customized to the instrument or parameter being assessed. Studies that attempted to probe energy deposition effects and reproducibility have been done with other classes of molecules, including drugs, peptides, and carbohydrates [8, 9, 36, 37]. However, none of these molecules have the specific combination of properties, described above, that make the benzylpyridinium-based thermometer ion species particularly useful as probes of MS performance.

In this study, we characterize the use of benzylpyridinium ions and their derivatives as probes of the ion production, electrochemical effects, energy deposition, ion transmission, and ion detection occurring within multiple ESI time-of-flight (TOF) mass spectrometers. From this we propose how mixtures of thermometer ions can be used to characterize the intensity reproduc- 
ibility of spectral profiles, and how instrument-based drift in the profiles can be detected and corrected automatically. These findings are a significant and practical step towards achieving analytical reproducibility of MS platforms in the intensity axis for biomarker discovery and other applications.

\section{Experimental}

\section{Materials}

The thermometer molecule 4-cyano-benzylpyridinium chloride (1) was received as a gift from the R. Graham Cooks laboratory at Purdue University. The remaining thermometer molecules [4-methoxybenzyl pyridinium chloride (2), 4-chlorobenzyl pyridinium chloride (3), and 4-nitrobenzyl pyridinium bromide (4)] were custom synthesized by GL Synthesis (Worcester, MA). All thermometer molecules were used without further purification.

Thermometer molecules were initially dissolved in methanol (Burdick and Jackson, Morristown, NJ) to yield a $1 \mathrm{mg} / \mathrm{mL}$ solution. These stock solutions were subsequently diluted to a working concentration using $90 \%$ aqueous acetonitrile (Burdick and Jackson or EM Science) / 0.2\% formic acid (Pierce Chemical, St Louis, $\mathrm{MO}$ ) containing $5 \mathrm{mM}$ ammonium formate (wt/vol) (Fluka, St Louis, MO). The final concentration of thermometer molecules in the working solution was $0.2 \mu \mathrm{M}$ for the JEOL MS system (Tokyo, Japan) and $0.05 \mu \mathrm{M}$ for the Agilent MS system (Santa Clara, CA).

\section{Serum Source, Protein Depletion, and Analysis}

Human serum samples were acquired from ProMedDx (Norton, MA) and were stored at $-80{ }^{\circ} \mathrm{C}$ until used. Sera were prepared for MS analysis by an initial tenfold dilution in $50 \mathrm{mM}$ ammonium formate buffer ( $\mathrm{pH}$ 6.5) and subsequent protein precipitation using a 9-fold excess of $90 \%$ aqueous acetonitrile containing $50 \mathrm{mM}$ ammonium formate. Samples were then placed in a centrifuge and spun at $3000 \times g$ for 15 min after which a $75 \mu \mathrm{L}$ aliquot of the supernatant was transferred into a microtiter plate and diluted 5-fold by the addition of $80 \%$ aqueous acetonitrile containing $0.25 \%$ formic acid ( vol/vol).

Processed serum samples were placed in all wells of Columns 3 through 12 of the microtiter plate. Columns 1 and 2 of the plates were filled with a buffer blank and a thermometer ion solution, respectively. Two plates were prepared and analyzed each day for 3 consecutive days. The analysis was performed on the Agilent LC/ TOF using the default instrument conditions.

\section{Instrumentation}

MS analysis was performed on three instruments from two MS instrument vendors: two JEOL JMS-T100LP orthogonal-TOF liquid chromatography-MS (LC/MS) systems from the same manufacturing batch; and an Agilent orthogonal TOF LC/MS model 6210 system. The instruments were used as installed by the respective manufacturers, except that an Alicat Scientific (Tucson, AZ) model no. MC-5SLPM-5D mass flow controller was added to the JEOL instruments to more precisely control the nebulizer gas flow. The mass flow controller was connected between the existing ball-valve control and the spray needle, improving the accuracy and precision of the gas control over 5-fold. Both brands of instrument are equipped with an analog-to-digital (ADC)-based digitizer for extended dynamic range.

All experiments were performed in the positive ion mode. The $m / z$ axis was calibrated according to the manufacturer's recommendation, using a mixture of polypropylene/polyethylene glycol for the JEOL instrument and using a mixture of phosphazines for the Agilent instrument. Default operating conditions were arbitrarily defined as those settings initially recommended by the vendors at the time of installation, which were expected to be optimal for the intended application of serum analysis. Default parameters for the two instruments were as follows:

For the JEOL, data were acquired from $\mathrm{m} / \mathrm{z} 100$ to 1000 at a rate of 1 spectrum every 2 s. Samples were introduced into the MS system using an Agilent 1100 or 1200 LC system configured with a binary pump, a micro well-plate autosampler, and a Peltier cooler set at $4{ }^{\circ} \mathrm{C}$. The LC flow rate was set at $50 \mu \mathrm{L} / \mathrm{min}$ and the mobile phase consisted of $90 \%$ aqueous acetonitrile with $0.2 \%$ formic acid (vol/vol). Samples were introduced using a partial loop injection of $40 \mu \mathrm{L}$. Default source parameters were spray voltage, $1900 \mathrm{~V}$; orifice 1 voltage, $40 \mathrm{~V}$; ring lens voltage, $10 \mathrm{~V}$; orifice 2 voltage $5 \mathrm{~V}$; peaks (rf) voltage, $1000 \mathrm{~V}$; and drying gas, $250{ }^{\circ} \mathrm{C}$.

For the Agilent, data were acquired from $\mathrm{m} / \mathrm{z} 100$ to 1650 at a rate of 1.35 spectra per second. Samples were introduced into the MS system using an Agilent 1200 LC system configured with an isocratic binary pump, a micro well-plate autosampler and a Peltier cooler set at $4{ }^{\circ} \mathrm{C}$. The LC flow rate was set at $100 \mu \mathrm{L} / \mathrm{min}$ and the mobile phase consisted of $90 \%$ aqueous acetonitrile with $0.2 \%$ formic acid (vol/vol). Samples were introduced using a partial loop injection of $80 \mu \mathrm{L}$. Default source parameters for the Agilent instrument were: capillary (spray) voltage, $3200 \mathrm{~V}$; fragmentor voltage, $165 \mathrm{~V}$; skimmer voltage, $65 \mathrm{~V}$; octapole rf voltage, $250 \mathrm{~V}$; and drying gas, $300^{\circ} \mathrm{C}$.

\section{Source Temperature Experiments}

In experiments involving changes in source temperature settings (i.e., drying gas) thermometer ion solutions were infused into the bulk solvent flow from the LC system using a tee placed just before the inlet to the mass spectrometer ion source. The concentration of the thermometer ion solution in the syringe was adjusted to yield a final concentration in the bulk solvent flow of 0.2 $\mu \mathrm{M}$ for the JEOL or $0.05 \mu \mathrm{M}$ for the Agilent. These 
concentrations were chosen based on experiments designed to find equivalent conditions that achieve good signal-to-noise on each system while minimizing any risk of saturating the detection system. Source temperatures were found to equilibrate in $\sim 5 \mathrm{~min}$, so an equilibration time of $10 \mathrm{~min}$ was allowed between data acquisitions each time the source temperature was changed to ensure complete temperature stability. The temperature ranges explored on each instrument spanned the default setting. The maximum and minimum values were determined by either the limit of accessible temperature or by the limit of spray stability.

\section{Spray Needle Position Experiments on the JEOL Instrument}

The spray needle adjustment knob on the JEOL was used to adjust the position of the spray needle in $\sim 1$ $\mathrm{mm}$ increments. The position adjustment started from the default position and first lowered the needle. Then the default position was restored and the experiments were repeated by raising the needle. The thermometer molecule sample was continuously infused for the length of the entire experiment, with $1 \mathrm{~min}$ of data acquired at each step, to generate each spectrum. Data acquisition at each step was initiated after the spray had stabilized.

\section{Data Analysis}

Loop injection of the samples yielded a broad injection profile with a flat plateau yielding a stable signal of $\sim 40$ $\mathrm{s}$ duration. For data analysis, $30 \mathrm{~s}$ of spectra were extracted from the middle of the plateau and summed to reduced minor between-spectrum fluctuations and improve the signal-to-noise ratio. Peak intensities were determined from the apex of the intact thermometer ion species (observed without the corresponding salt counter ion) and their corresponding fragments. For simple analyses of the effect of single parameters on energy deposition and ion transmission of a single thermometer ion, ratios of the intensities of the precursor $(\mathrm{P})$, and their corresponding fragment ion(s) (F) were used. For comparisons of instrument effects across multiple individual thermometer ions or instruments, it was useful to have a normalized representation of the thermometer ion behavior (since P:F ratios vary so widely); therefore the results are presented as a survival yield $(S)$ according to eq 1.

$$
S=\frac{P}{\left(P+\sum_{1}^{i} F\right)}
$$

Where,

$S$ is the survival yield value

$P$ is the intensity of the precursor molecular ion

$F$ is the intensity of the benzyl fragment ion plus any related fragments.
To facilitate a simplified evaluation of the impact of instrument tuning changes on several thermometer ions simultaneously, a change in the values of the survival yield of Compounds $\mathbf{1}$ to $\mathbf{4}$ was represented as the change in the Euclidean distance between two points in a 4-dimensional space. The coordinates determined from the four individual survival yields, obtained under a given set of instrument operating conditions, defined a point in this four-dimensional space. The reference point from which distances were measured was generated from the average survival yield of each of the four compounds determined from eight replicate measurements of a mixture of the four thermometer ions under the "default" instrument conditions.

\section{Results and Discussion}

\section{Instrument Configurations}

Two orthogonal TOF instruments were used in this work that have different ion source configurations. The Agilent 6210 LC/TOF instrument employs a capillary (referred to as the fragmentor) to transmit ions between two different pressure regimes of the ion source. The JEOL JMS-T100LP AccuTOF employs a series of skimmers (referred to as orifices 1 and 2) to accomplish the same goal. The JEOL instrument has more ion optical elements before the radio frequency (rf) ion guide (i.e., in the higher pressure region of the source), consequently tuning on this instrument is more flexible but also more complex. Schematics of the Agilent and JEOL instrument ion sources are shown in Figure 1. The primary focus of this work was on the higher pressure regions of the ion sources, including ion optical elements up to the rf ion guide. The effect of the fragmentor or skimmers on the thermometer ions mirrors what has been described previously for other instrument

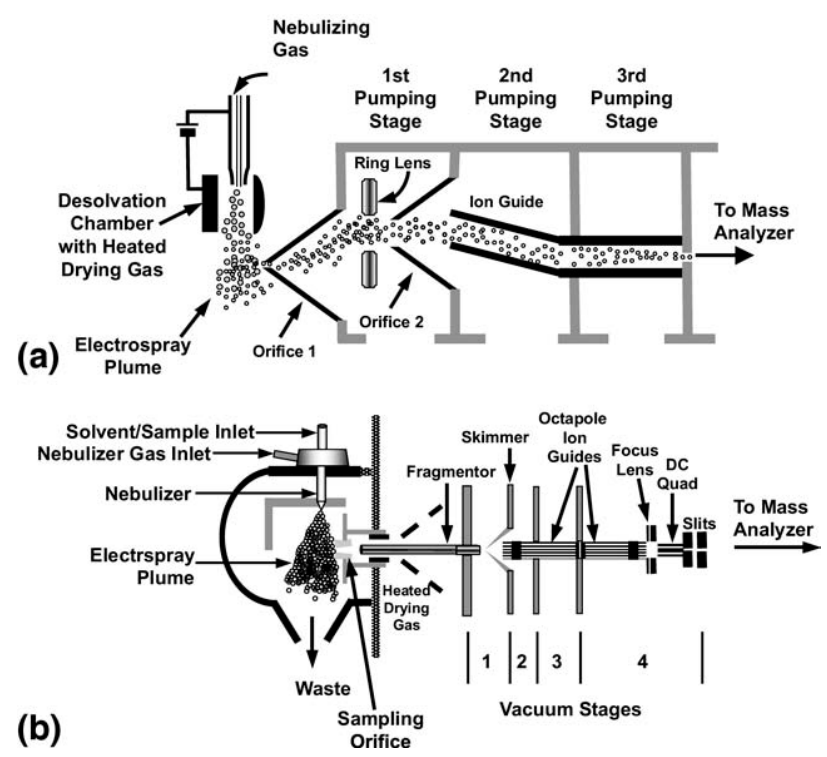

Figure 1. Simplified schematic view of the JEOL JMS-T100LP AccuTOF (a) and Agilent 6210 LC/TOF (b) source optics. 
types and therefore are not described in detail herein [28].

The general behavior of the thermometer ions in the Agilent and JEOL instruments is the same. As the energy deposited into the thermometer ions is increased, they undergo more fragmentation. The degree of fragmentation within each brand of instrument follows the same order for the thermometer ion series (Compound $2>3>1>4$ ), but the extent of fragmentation, for a given compound, differs substantially. Generally, the data presented below are limited to the specific thermometer ion or ions that yielded the best signal-to-noise for both the precursor and fragment peaks (dependent on both the experiment and instrument).

\section{Ion Production}

The mechanism of ion production $[38,39]$ in ESI is still a matter of debate $[40,41]$ although some recent work with benzyl pyridinium thermometer ions supports the charge residue model [42]. Regardless of the mechanism, the electrostatic dispersion of the solvent stream is achieved by a different application of the potential in the two instruments used in this study. The JEOL applies a high positive voltage to the spray tip and maintains the sampling orifice at a potential near to ground relative to the spray tip. Conversely, the Agilent maintains the spray tip at ground potential and has the sampling orifice at an "elevated" negative potential. Additionally, the spectra of the thermometer ions are different for the two systems, in that JEOL spectra, like those derived from some other instruments [43], exhibit acetonitrile solvent adducts of the benzyl fragments, in addition to the expected fragmentation shown in Scheme 1. Whether the difference in electrical configuration is the source of the difference in the production of the adducts is unclear, but variable production of such adducts across instrument types has be reported before [43].

The amount of the adduct generated is often significant (Figure 2) and varies with the para-substituent (Table 1). A survival yield analysis [44] gives different survival curves, depending on whether the adduct ion is included as a fragment or not. It is not unreasonable to anticipate that similar adduct formation could occur with other molecules. Therefore, given the complexity of most biological samples and the consequent risk of inadvertently modifying the samples, it would seem that instrument configurations that avoid this phenomenon would be preferable for such analyses.

The intrinsic charge on the thermometer ions and their simple fragmentation yield reasonable repeatabil-

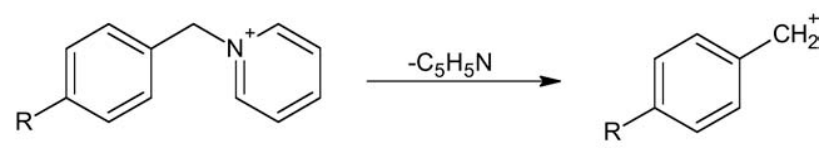

Scheme 1. $1 \mathrm{R}=\mathrm{CN} ; 2 \mathrm{R}=\mathrm{OCH}_{3} ; 3 \mathrm{R}=\mathrm{Cl} ; 4=\mathrm{NO}_{2}$.

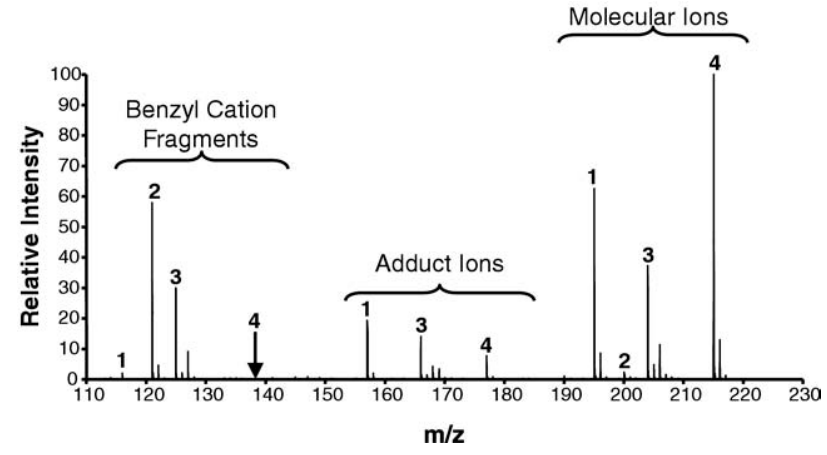

Figure 2. A Spectrum of four different thermometer ion species on the JEOL AccuTOF. Molecular and fragment ions from the various thermometer ion species are indicated by the corresponding compound numbers. The JEOL instrument generates solvent adducts at $\sim 41 \mathrm{Da}$ above the expected thermometer ion fragments.

ity when measuring the precursor:fragment peak intensity ratio (P:F), even when the analysis is interleaved with serum samples (Figure 3a). In the experiment in Figure $3 a$, the instrument was operated continuously for 3 days, while analyzing protein precipitated serum samples (i.e., "dilute and shoot" samples), arguably a worst-case scenario, yet the \% CV in the P:F ratio was $20.5 \%$. This result suggests that the P:F may be a reasonable general measure of instrument reproducibility over longer periods of time or of reproducibility between instruments.

The degree of fragmentation of the thermometer ions differs depending on their para-substituent and by instrument type; in this case the JEOL inducing greater fragmentation than the Agilent (e.g., Figure $3 b$ and c). The ability to "select" the level of observed fragmentation by varying the substitution on the benzyl moiety offers several advantages. First, thermometer ions that fragment to the desired level (i.e., yield good signal-tonoise for both precursor and fragment ions under the chosen experimental conditions) can be selected for different instrument configurations. Second, by choosing a range of thermometer ion species that fragment at different internal energies, the impact of the ion optics further into the optical system can be probed (thermometer ions that are largely fragmented in the early stage of the source provide little information on the later stages). In this case the difference in the relative survival yield of different thermometer ion species across different stages of the atmosphere vacuum interface may be used as a probe of differences in both energy deposition and ion transmission.

\section{Electrospray Conditions}

Changing the spray tip voltage through a small range close to the default voltage (optimized for reserpine sensitivity) does not change the degree of fragmentation for the thermometer ion species for either instrument. However, the position of the spray tip relative to 
Table 1. Relative fragment ion intensities for Compounds $\mathbf{1}$ to 4

\begin{tabular}{|c|c|c|c|c|c|}
\hline Compound & $\begin{array}{c}\text { Para } \\
\text { substituent }\end{array}$ & $\begin{array}{l}\text { Fragment ion } \\
\text { rel. }^{a} \text { intensity }\end{array}$ & $\begin{array}{l}\text { Adduct ion } \\
\text { rel. intensity }\end{array}$ & $\begin{array}{l}\text { Precursor ion rel. } \\
\text { intensity }\end{array}$ & $\begin{array}{c}\text { Adduct ion:fragment } \\
\text { ion ratio }\end{array}$ \\
\hline 2 & $\mathrm{p}-\mathrm{OMe}$ & 57.9 & $n / d^{b}$ & 2.4 & $\mathrm{n} / \mathrm{a}$ \\
\hline 3 & $\mathrm{Cl}$ & 30.0 & 14.0 & 37.3 & 0.5 \\
\hline 1 & $\mathrm{CN}$ & 2.0 & 19.3 & 62.4 & 9.7 \\
\hline 4 & $\mathrm{NO}_{2}$ & 0.3 & 7.7 & $100^{c}$ & 25.7 \\
\hline
\end{tabular}

arelative.

bNot detected.

'Base peak in the spectrum.

the sampling orifice (Figure 3d) on the JEOL instrument does have an impact. As demonstrated for Compound 3 , the observed P:F changed as the relative position between the spray tip and orifice 1 is changed. Additionally, the degree of variability in the ratios between replicates increases as the spray tip position is moved further from the default position. At the lowest needle position, the spray was not sufficiently stable to yield a reliable signal and no measurement was attempted. A similar phenomenon with respect to P:F ratios was observed by Nefliu et al. [29] in the case of desorption electrospray ionization (DESI) and electrosonic spray ionization (ESSI).

In contrast, the spray tip position on the Agilent system is fixed relative to the sampling orifice so comparable experiments cannot be performed. While the ability to adjust the spray tip position allows the operator to optimize performance for a variety of solvent systems and flow rates, this may not be optimal from the point of view of reproducibility of data across multiple experiments or instruments. Given the variable impact on the energy deposition in the analyte ions, a precise mechanism for ensuring the spray tip is in the same position across those experiments is essential. However, it is not clear that simply maintaining a constant distance between the spray tip and the ion sampling orifice will yield equivalent results across instruments of different types, given the likely differences in the gas dynamics, energy deposition, and ion transmission effects on instruments from various vendors. One potential solution is to establish a quality control and adjustment based on historic performance of appropriate thermometer ions and use this to qualify needle settings before use.

Other factors that have been reported to have an impact with respect to the electrospray are the solvent flow rate and the nebulizing gas flow. Solvent flow rates can be well controlled by an LC system and gas flows can similarly be well controlled, if necessary by a mass flow controller, as was done for the JEOL instrument. These parameters were therefore not characterized for their effects on reproducibility in this work.

\section{Source Temperature}

The temperature of a resistively heated capillary used to transmit ions from atmospheric pressure into the first stage of the vacuum affects fragmentation [34]. As the temperature increases, it is thought that the small electrospray droplets evaporate more quickly, before the final stage of desorption and ionization. Therefore, the collisions between the essentially desolvated ions in the region between the capillary and the skimmer impart energy into the analyte rather than completing the desolvation by collisional declustering.

Both the JEOL and Agilent instruments can heat the incoming electrospray droplets, although they achieve it differently. The JEOL instrument applies a heated "drying gas" to the electrospray plume at atmospheric pressure, before the analyte enters the sampling orifice (i.e., orifice 1). The Agilent system has a heated gas flow between the sampling orifice and the fragmentor, which indirectly heats the fragmentor and neighboring elements. Nevertheless, in each of these systems, the degree of fragmentation of the thermometer ions exhibits a temperature dependence, as demonstrated for Compound 2 in Figure $3 \mathrm{~b}$ and c (the other thermometer ions show a similar trend). The relative change in the thermometer ion peak ratios is greater for the Agilent instrument, implying that either the heat transfer is greater in this instrument configuration or that the analyte ions started from a higher degree of desolvation. In either case, the applied heat leads to changes in energy deposition in the thermometer ions.

These temperature-dependent results highlight yet another parameter that should be carefully monitored, controlled, and standardized across experiments to ensure reproducible results. While that is, in principal, straightforward to achieve on a single instrument type, it is less clear how it may be achieved across different instrument platforms that employ different heating strategies. Simply setting the relevant software control to the same temperature setting does not ensure that the same degree of heating is achieved by the different heating configurations. The work of Collette et al. and others $[24,26,45]$ to characterize the temperature of the thermometer ions therefore becomes of practical utility in estimating the impact of ion source temperature with respect to its influence on the reproducibility of spectra. However, a simpler strategy than fitting the data from multiple thermometer ion species to a Boltzmann distribution might facilitate its use in routine analysis of analytical samples. Since the specific temperature of the ions is less of an issue than temperature variation, the 

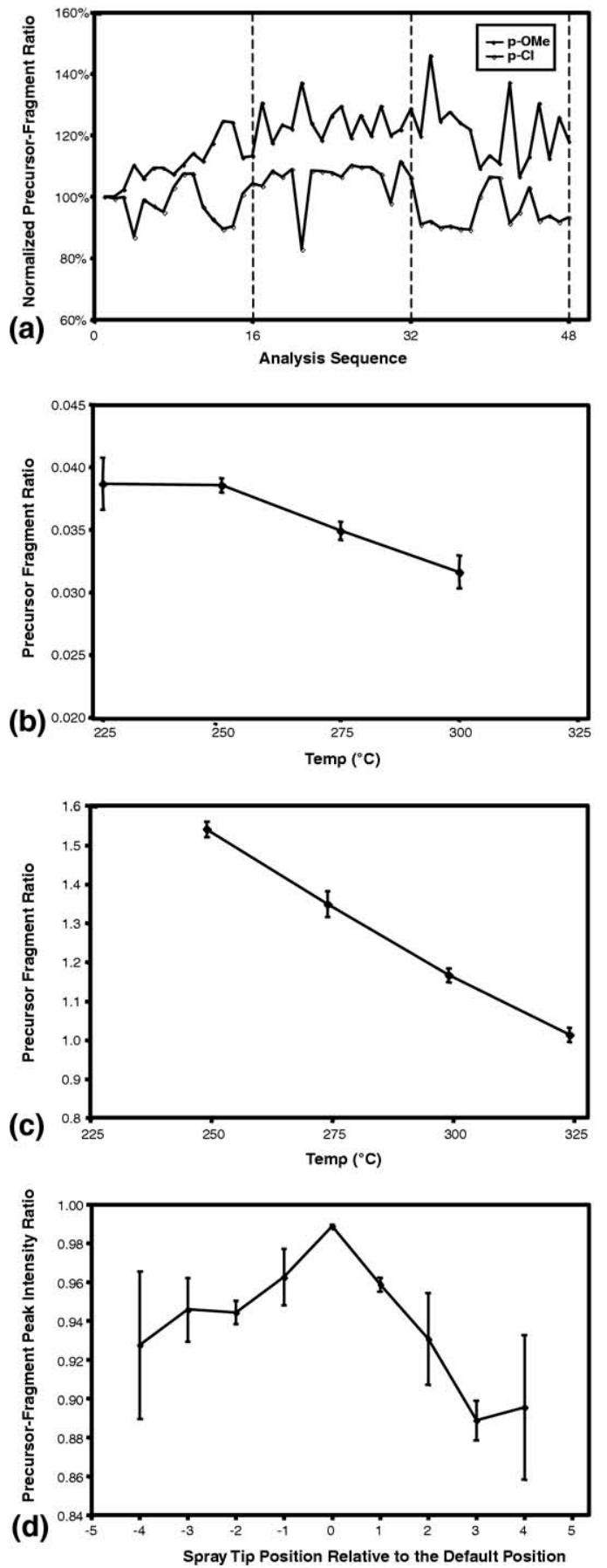

Figure 3. Repeatability of thermometer ion precursor fragment peak intensity ratio measurement over three days of continuous analysis (a); effect of drying gas temperature on the precursorfragment peak ratio of the p-methoxy benzylpyridinium thermometer ion: JEOL (b), Agilent (c); variability of the energy deposition in the thermometer ions: dependency on the JEOL AccuTOF spray tip position (d).

difference in the precursor-fragment intensity ratio (or survival yield) of one or a few selected thermometer ion species should suffice to standardize the source temperature, to ensure uniformity across experiments, as well as stability within experiments. Separate thermometer ion species could then be used to control for other variables in the ion source. In such a strategy, however, it is critical that the system is completely equilibrated with respect to temperature before experiments are begun. This includes ensuring that an LC system has been flowing solvent into the instrument, since the solvent load can itself affect the final equilibrated temperature.

\section{rf Ion Guides}

Source conditions can influence both the qualitative and quantitative aspects of MS/MS data on instruments employing a quadrupole collision cell [28]; so any perturbations to ion internal energy in the early stages of an ion source have an impact further downstream. By analogy to a quadrupole collision cell, many current commercial API ion sources employ an rf ion guide to focus and transmit ions from one region of the source to another [46], so it might be reasonable to expect additive effects on ion internal energy to be general to such API ion sources.

Similar to a collision cell, fragmentation can be induced in an rf ion guide of a orthogonal TOF instrument [47]. So, if ions have previously been heated but not fragmented in the upstream regions of the ion source, a relatively small additional increase in translational energy can induce fragmentation in the rf ion guide as shown in Figure $4 \mathrm{a}$ and $\mathrm{b}$. It is clear from the plot that a combination of lower fragmentor voltage and a higher skimmer voltage can achieve the same degree of fragmentation as a high fragmentor voltage alone. Similar trends are observed for the adjustment of various combinations of the orifice 1 or 2 and ring lens voltages on the JEOL instrument. However, the added complexity of the tuning space prevents a simple presentation of the data (e.g., Figure $4 a$ and b).

\section{Ion Transmission}

While energy deposition is important to spectral content, $m / z$-dependent transmission effects also have a significant impact on observed spectral peak intensities. Consequently, calibration point strategies for tuning instruments [17] to yield similar spectral content may sometimes be inadequate to achieve the desired level of similarity in y-axis reproducibility.

Instruments using a quadrupole rf ion guide, like the JEOL AccuTOF, have a relatively well-defined fall-off in transmission, at low $m / z$, at some rf voltage settings (Figure 5a). Generally, for a fixed frequency, a higher rf voltage will improve transmission for higher $m / z$ species [47], but there is a decrease in the transmission of lower $\mathrm{m} / \mathrm{z}$ ions, as indicated by the higher precursorfragment $(\mathrm{P}: \mathrm{F})$ peak ratio in Figure $5 \mathrm{a}$ as the rf voltage is increased. These, rf-dependent transmission characteristics vary between the two JEOL instruments used in this study (Figure 5a and b). There is a difference in both the voltage at which there is a rapid change in the P:F peak ratio, and the value of the P:F ratio itself, 

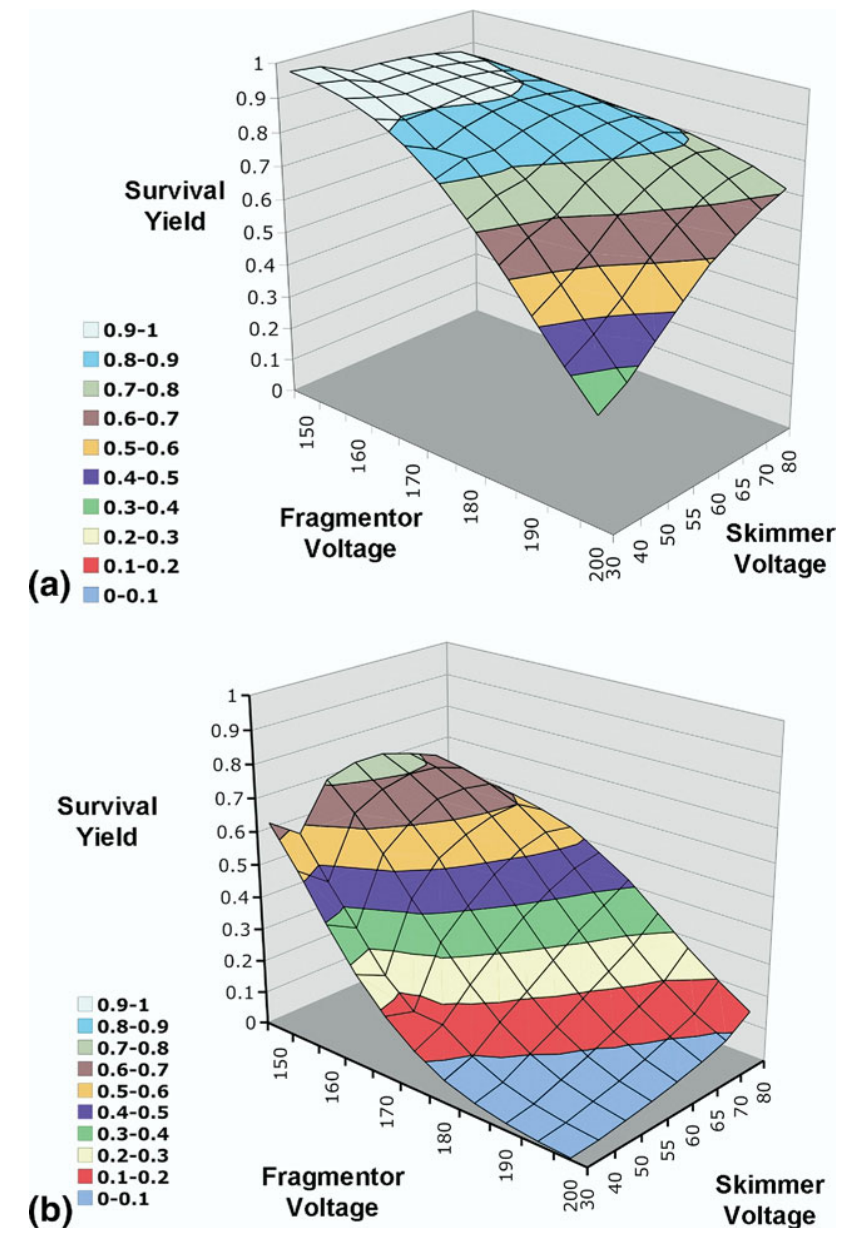

Figure 4. Tuning surface survival yield plots. Survival yield surface plot for the (a) p-Chloro- and (b) p-methoxy benzylpyridinium thermometer ions on the Agilent system. The two surfaces yield complementary information.

although there is a region of "stability" on both instrument below $\sim 900 \mathrm{~V}$. However, a typical rf voltage setting on the AccuTOF for optimal sensitivity in the range above $\mathrm{m} / \mathrm{z} 1000$ would be $\sim 1500 \mathrm{~V}$. This instrument-dependent difference could make comparison of the data from two instruments problematic. In contrast, octapole ion guides, like that used in the Agilent LC/TOF, exhibit less variation in $\mathrm{m} / \mathrm{z}$ dependent transmission of the thermometer ion precursor and fragment until a very low $\mathrm{rf}$ voltage setting, relative to the normal operating range (Figure 5c).

There are also more subtle effects that impact quadrupole-based ion guides. These guides exhibit transmission "nodes", narrow $\mathrm{m} / \mathrm{z}$ regions in which there is a significant variation in ion transmission relative to a neighboring $m / z$, as shown in Figure 5 b. As the rf voltage is adjusted, the specific $m / z$ that is affected changes. Both the magnitude of the effect and its position differs between the two JEOL AccuTOFs used in this study. Figures $5 a$ and $b$ are derived from changes in precursor fragment peak intensity ratios for the thermometer ion with the para-chloro substitution.
Given the possibility that the fragmentation might be driven by changes in the amplitude of the rf induced oscillations [47] isotopic peak ratios were also examined. As shown in Figure $5 \mathrm{~d}$, the relative ratio of the chlorine isotope peaks in Compound 3 (which should be constant) are also affected by changes in the quadrupole rf. This result implies that even biomarker studies which employ isotopic labeling strategies (e.g., ICAT, ITRAQ, AQUA) can be subject to this artifact, and consequent misleading quantification, if the impact of quadrupole rf voltage settings are not considered carefully.

\section{Ion Detection}

Saturation of the detection system can lead to the distortion of high abundance peaks in a spectrum, thereby affecting relative peak intensities. This effect can be pronounced for instruments employing time-todigital converter (TDC)-based signal digitizers [48, 49], although this has become less of a concern as the dynamic range of the instruments has increased with the use of ADC-based digitizers. However, a more subtle, yet equally critical, effect impacts peaks of low abundance during the detection/digitization process. The observed baseline in the digitized output is arbitrary, with the zero reference for the detector system being adjustable electronically. To improve the apparent signal-to-noise in spectra, on some spectrometers such as the Agilent and JEOL, an autotune or manual control can be used to adjust the digitizer baseline offset, and trim away some of the chemical and/or electronic baseline noise. If this adjustment is overly aggressive, a significant part of the signal may be excluded from the final spectrum, leading to a significant change in the apparent peak intensity ratio of small peaks. The impact on the chlorine isotope peak ratio of Compound 3 is illustrated in Figure 6 for a wide range of amplifier offset values. The range of settings generally obtained from the autotuning process is indicated in the same figure (area in circles). In this range of autotune-derived amplifier offset settings on the Agilent system, the relative change in the apparent chlorine isotope ratio is relatively small at $\sim 5 \%$. By comparison, the relative change in the peak intensity ratio between the monoisotopic peak and the adjacent peak in the isotope cluster is significantly larger, at $\sim 9 \%$. Thus, the effect of the baseline offset is magnified for peaks of lower absolute intensity. Furthermore, since these peaks are natural isotopomers of each other, the threshold effect of electronic offsets in the digitized signal will also skew the results from studies that use stable isotope-based strategies for biomarker discovery. Since many biomarkers of interest, especially in serum, are of relatively low abundance, the effect of changing electronic thresholds, through autotune functions in particular, might often have a significant impact when data are compared across experiments. 

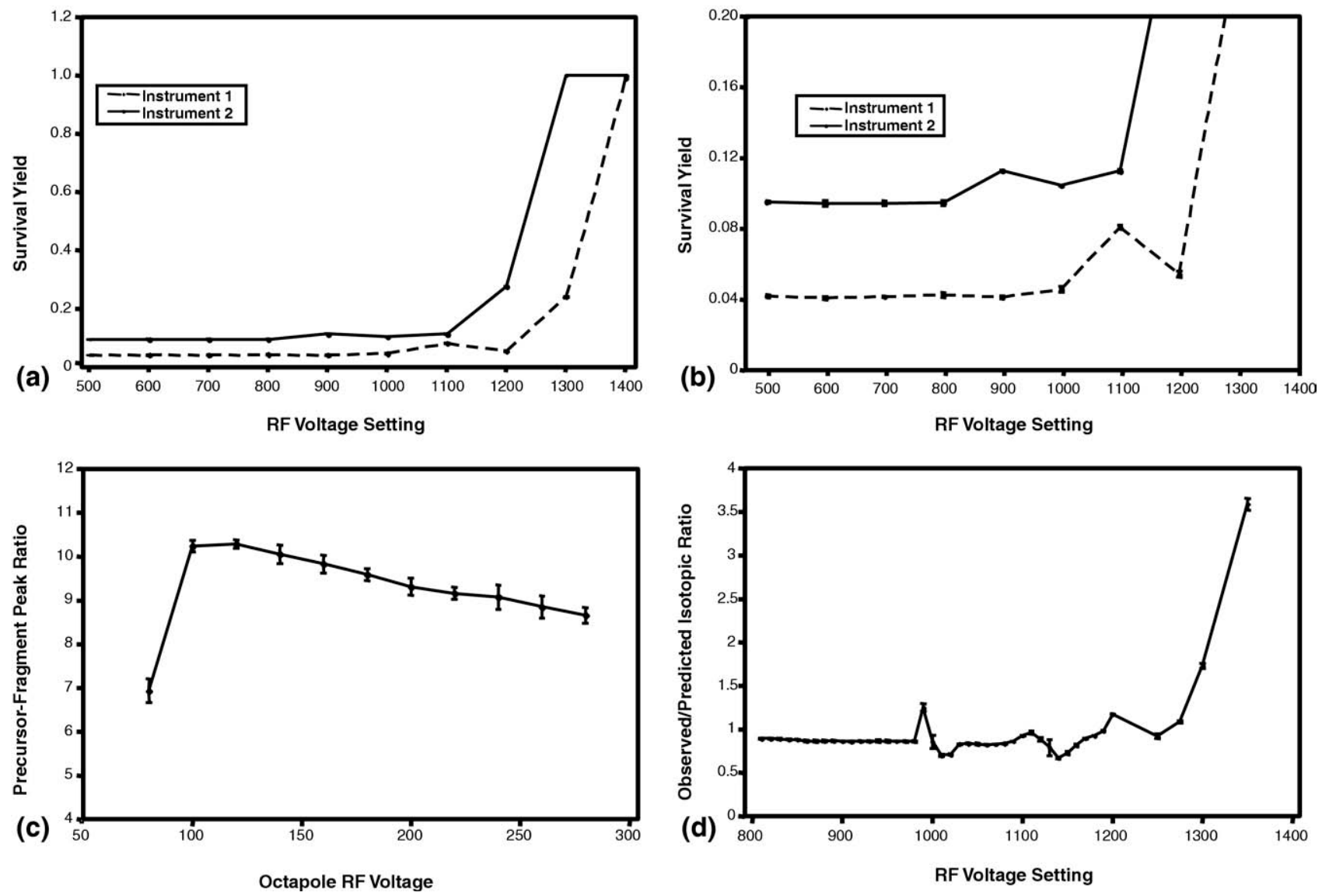

Figure 5. Low $\mathrm{m} / \mathrm{z}$ Effects of the Peaks (rf) voltage on the JEOL AccuTOF: (a) the low $\mathrm{m} / \mathrm{z}$ discrimination effect of changing the $\mathrm{rf}$ ion guide voltage. (b) An expansion in which the localized effects of the nodes are visible. On one instrument the effect of the node causes as much as a 2 -fold change in the observed peak ratios. (c) There is relatively little change in the P:F ratio as the rf voltage changes on the Agilent. (d) The normalized ratio of the observed versus predicted isotope ratios [ $(\mathrm{m} / \mathrm{z}$ 127 versus $\mathrm{m} / \mathrm{z}$ 125)/0.326] for the chlorine containing benzyl fragment ion of the $\mathrm{p}-\mathrm{Cl}$ thermometer ion species are significantly affected by small changes in the rf ion guide voltage. At low rf voltage the ratio is slightly less than theoretical, possibly because of signal saturation, whereas at higher voltages there is a differential discrimination against the ${ }^{35} \mathrm{Cl}$-containing isotopomer at $m / z 125$.

\section{Thermometer Ion-Based Strategy for Instrument Tuning}

Biomarker studies inherently involve the analysis of complex biological samples. Assessing the reproducibility of hundreds to thousands of features in these datasets is a problem involving very high dimensionality. Unlike MSbased pharmacokinetic studies, for example, in which one or a few molecules can be readily evaluated by plotting a simple two-dimensional chromatogram, biomarker studies require a more sophisticated representation of the data to capture the variability of the many chemical entities that are analyzed. Many factors will affect the reproducibility of the final data before the sample ever reaches the MS system for analysis. However, those factors are distinct from the question of dealing with the variability resulting from changes in the platform performance, which is the focus of this study.

Prakash et al. [12] presented an elegant approach to characterizing the variability in data derived from
LC/MS experiments. Nevertheless, their approach assesses the data after the fact, when any instrumental drift has already had its potential negative impact. A more proactive strategy is needed. Given the useful physicochemical properties of benzylpyridinium thermometer ions and their ability to sensitively probe multiple aspects of instrument performance, we investigated how mixtures of these molecules could be used to evaluate changes in instrumental performance before sample analysis and then used to compensate for observed changes.

To assess multiple aspects affecting instrument performance simultaneously, mixtures of thermometer ion species can be analyzed with negligible interference with each other [24, 29]. Taking advantage of the variable, but predictable, stability of different thermometer ion species, mixtures can be designed in which different ions fragment primarily within different regions of the ion source, depending on the specific 

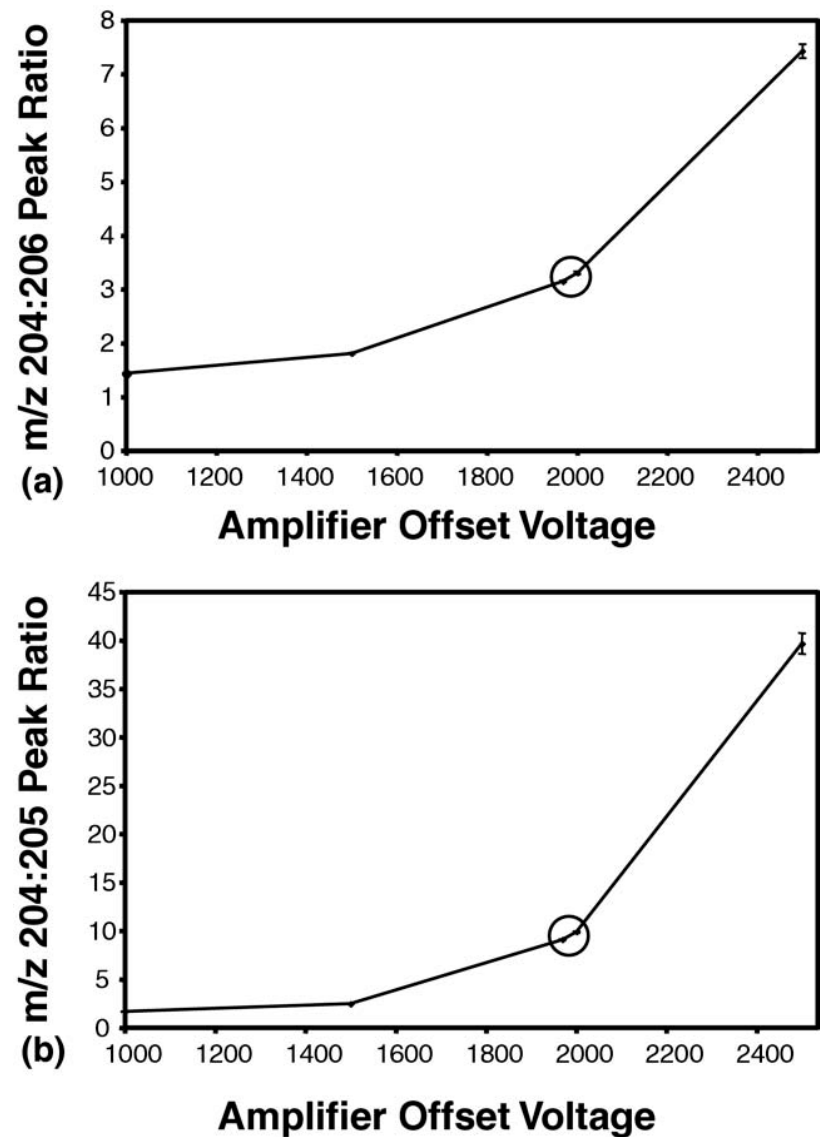

Figure 6. Amplifier Offset Parameter Effects on Observed Peak Intensity Ratios. In (a) the effect on the chlorine isotope peak ratios is plotted. In (b) the effect on the monoisotopic versus $13 \mathrm{C}$ isotope peak ratio is plotted. As expected, the effect is larger on the smaller peak. The circles indicate the normal operating range used in these studies.

source conditions. Consequently, a mixture of these species could be used to probe multiple aspects of an API ion source in a single analysis and their data used to monitor and, if necessary, adjust for instrument drift.

Since multiple thermometer ions would be used to monitor a variety of source factors, a simplified representation of the data would be useful, especially for automation of the process. One useful way of presenting these data is by defining a reference point in a multi-dimensional space based on the intensity of various ions in a reference mixture [50]. For example, using a mixture of three species, one could define a reference point in a three-dimensional space, with the intensity of each molecular ion representing one coordinate in that space. Using that reference mixture to measure instrument performance at some future time would generate a new point in the three-dimensional space. Instrument drift is then monitored as a change in the Euclidean distance $\left(\right.$ distance $\left.=\left(\Delta \mathrm{x}^{2}+\Delta \mathrm{y}^{2}+\Delta \mathrm{z}^{2}\right)^{1 / 2}\right)$ between the newly measured point and the predetermined reference point. Figure 7a schematically illustrates the concept for a three-component mixture in a three-dimensional space. Other measures of distance between the refer- ence point and subsequently measured test points, such as the Mahalanobis distance, which takes into account correlations in the data and is independent of the scale, can also be used [51].

In principal, such measurements could be made with any mixture of reference compounds [50]; however, repeated preparations of the reference mixture and differential chemical stability of it constituents would create their own variability in the analysis. Alternatively, by using thermometer ions that fragment internally, the data from each individual thermometer ion species can be expressed as a survival yield (S1, S2, $\mathrm{S} 3, \ldots \mathrm{S} n)$, rather than as a series of intensities. In turn, those survival yields, rather than the raw intensities, can be used to build a multi-dimensional reference point. This has several advantages. First, a survival yield (essentially a ratio measurement) is much less sensitive to between-batch variability in the concentration of the individual components of a reference mixture; every component is essentially normalized before use. Second, a survival yield-bases analysis is less sensitive to peak intensity changes caused by fluctua-

(a)
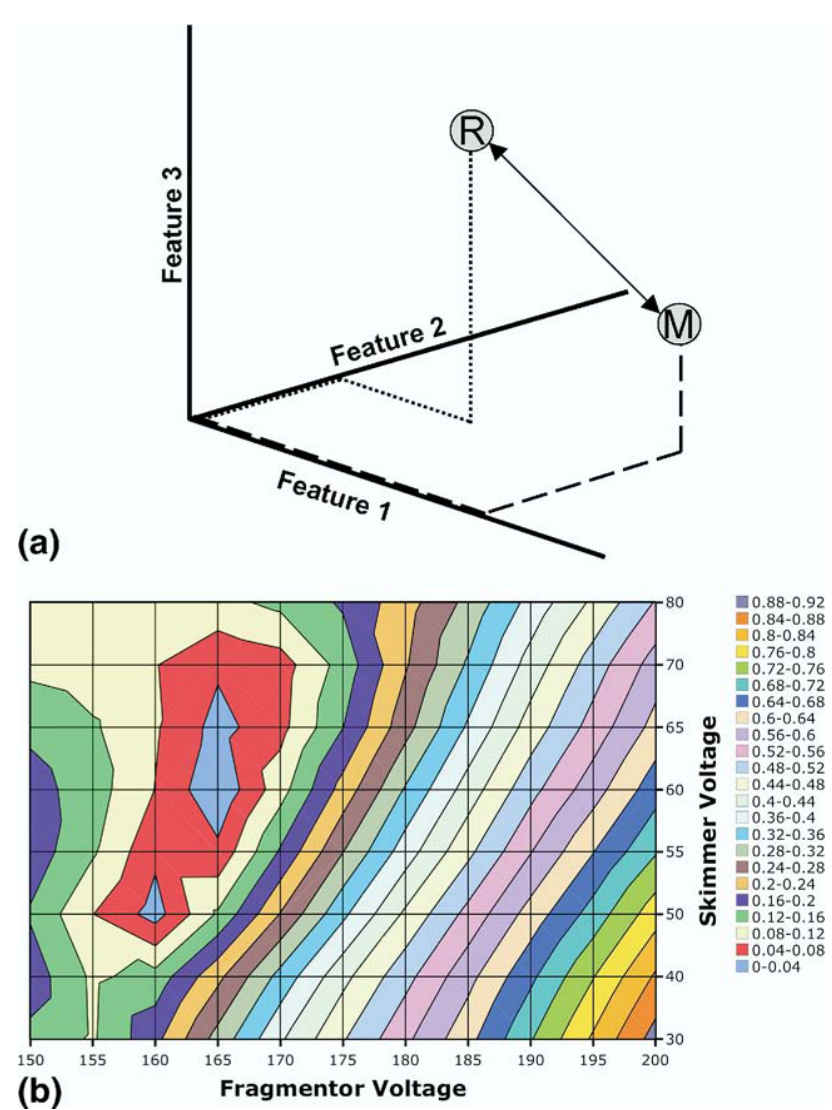

Figure 7. An exemplary three-dimensional plot is shown in which three features (i.e., biomarker peak intensities) define and the distance between a reference point $(\mathrm{R})$ and a measured tuning profile (M) (a). A tuning contour plot derived from thermometer ion-based distance variation: the reference point was determined from the survival value of four thermometer ions (Compounds 1-4) in eight replicate analyses under the default conditions (i.e., Fragmentor $165 \mathrm{~V}$ and Skimmer $65 \mathrm{~V})(\mathbf{b})$. 
tions in ionization, differential chemical stability of the reference compounds, small changes in buffer composition, and other factors that could affect raw peak intensities.

Figure $7 \mathrm{~b}$ illustrates a contour plot showing changes in the Euclidean distance between a reference point (derived from a mixture of thermometer ions analyzed under conditions in which the fragmentor voltage and skimmer voltage were varied on an Agilent LC-TOF relative to the "default" instrument parameters. There is clearly a range of conditions that yield comparable results, suggesting that small changes in the settings of instrument parameters need not always have an impact on spectral content. Also, within a limited range, a change in spectral content induced by a change in one parameter may be compensated for by an adjustment in another source parameter. This behavior may afford a means for retuning an instrument to compensate for drift over time as the front end of the source becomes contaminated by repeated sample analyses. We recently demonstrated [50] that a mixture of compounds can be used to retune an instrument to yield comparable spectral data from serum after a variety of routine maintenance procedures.

By analogy to the work of Bristow, Hopley and coworkers [9, 17], thermometer ion-based retuning might also afford a way to adjust multiple instruments to yield a similar spectral content. Furthermore, the surface defined by the changing in distance between the reference point and the newly measured results is relatively simple, at least in the case of the Agilent system. This suggests the possibility that retuning an instrument to minimize the difference between the reference point and current tuning may be automated. The case of the JEOL is more complex because of the higher dimensionality of the tuning space; however, a variety of existing algorithms for distance minimization could work to optimize the tuning on either instrument. Suitable algorithms might include, for example, a conjugate gradient or simplex algorithm [52]. To facilitate the tuning, the thermometer ion mixture could be infused into an instrument in the same way that tuning and mass calibration mixtures are employed; only, in this case, the goal would be to tune/calibrate the y-axis performance.

\section{Potential Future Directions}

One limitation to the specific mixture of thermometer ion species used in this study is that they were designed to have a similar collision cross section and mass so that those properties would not affect the estimation of their internal energy distribution [24, 26]. To the extent that mass or collisional cross section would be important to probe gas dynamic effects or other instrumental properties that impact spectral content, a more diverse mixture of thermometer ions species might be useful. Given the utility of having easily substituted benzyl moieties that control the observed fragmentation, pre- serving that portion of the molecules would be useful. Furthermore, the intrinsic charge on the molecules minimizes effects from competitive ionization; therefore, preserving the quaternary ammonium group is desirable. However, analogous thermometer ion species with suitable fragmentation pathways could readily be generated from a variety of native or substituted nitrogencontaining aromatics as illustrated in Scheme 2.

Future work will also require the comparison of a larger set of instruments and a more detailed correlation of the changes observed with thermometer ions with those obtained from biological samples. The later step is necessary to define suitable limits or tolerances for retuning or accepting the tuning of the instrument. Again, by analogy to tuning or calibrating the $m / z$ scale, when the mass accuracy or resolution is beyond specified criteria, the retuning/calibration procedure is executed. A similar strategy is envisioned for thermometerion based tuning of the $y$-axis.

The optimal mixture of thermometer ions also remains to be determined and might be instrumentdependent. To minimize the practical and computational complexity of implementing this methodology, the minimum number of thermometer ions necessary to account for the degrees of freedom in the system would be used. Since some aspects of the instrument performance have a bigger influence than others, only primary factors affecting y-axis reproducibility would be adjusted as part of the tuning process.

\section{Conclusions}

Benzylpyridinium salts can be used to probe multiple aspects of performance of an API-source equipped mass spectrometer, including temperature-based effects, energy deposition effects, transmission effects, and ion detection effects. They provide a sensitive measure of changes in the source conditions that can be used to define differences in instrument performance over time or differences in performance between two instruments of either the same or different type. The use of the Euclidean distance between multi-dimensional points defined by the survival yield of mixtures of thermometer ions appears to be a useful approach to representing the higher dimensional data that is necessary to

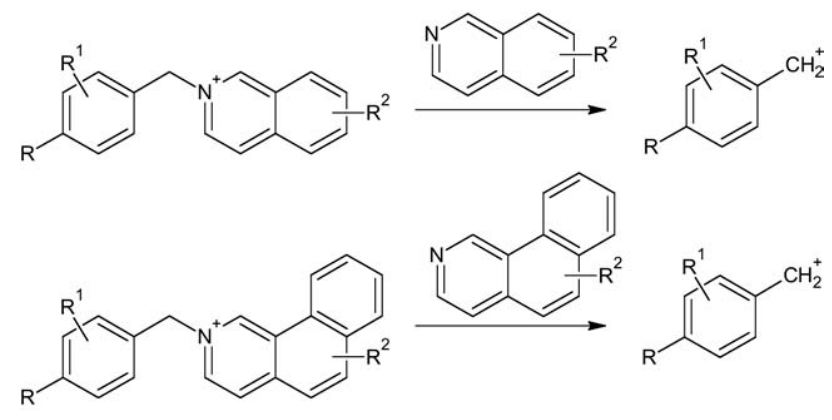

Scheme 2. $\mathrm{R}_{1}$ or $\mathrm{R}_{2}$ or $\mathrm{R}_{3}=\mathrm{O}$-alkyl or alkyl or $\mathrm{CN}$ or $\mathrm{NO}_{2}$ or halogen. 
define the performance effects of multiple instrumental factors and parameters. This approach may lend itself to automation, to facilitate establishing and maintaining uniform instrument performance within and between labs for biomarker studies, or other applications that require reproducibility in the intensity axis of mass spectral data.

\section{Acknowledgments}

The authors thank Mr. Jun Tamura of JEOL Ltd. for providing his detailed insights on the ion optics and general instrument performance of the AccuTOF. Additionally, they acknowledge Dr. David Weil of Agilent Technologies, Inc. for helpful advice in the initial tuning of the $6210 \mathrm{LC} / \mathrm{TOF}$. They also thank the R. Graham Cooks laboratory for the generous gift of the 4-cyano benzylpyridinium chloride. Finally, the authors thank Dr. Greg Bertenshaw for critical reading of this manuscript.

\section{References}

1. Ausloos, P.; Clifton, C. L.; Lias, S. G.; Mikaya, A. I.; Stein, S. E.; Tchekhovskoi, D. V.; Sparkman, O. D.; Zaikin, V.; Zhu, D. The Critical Evaluation of a Comprehensive Mass Spectral Library. J. Am. Soc. Mass Spectrom. 1999, 10(4), 287-299.

2. Han, D. K.; Eng, J.; Zhou, H.; Aebersold, R. Quantitative Profiling of Differentiation-Induced Microsomal Proteins Using Isotope-Coded Affinity Tags and Mass Spectrometry. Nat. Biotechnol. 2001, 19(10), $946-$ 951.

3. Ross, P. L.; Huang, Y. N.; Marchese, J. N.; Williamson, B.; Parker, K.; Hattan, S.; Khainovski, N.; Pillai, S.; Dey, S.; Daniels, S.; Purkayastha, S. Juhasz, P.; Martin, S.; Bartlet-Jones, M.; He, F.; Jacobson, A.; Pappin, D .J. Multiplexed Protein Quantitation in Saccharomyces cerevisiae Using Amine-Reactive Isobaric Tagging Reagents. Mol. Cell. Proteom. 2004, 3(12), 1154-1169.

4. Gerber, S. A.; Rush, J.; Stemman, O.; Kirschner, M. W.; Gygi, P. Absolute Quantification of Proteins and Phosphoproteins from Cell Lysates by Tandem MS. Proc. Natl. Acad. Sci. U.S.A. 2003, 100(12), 6940-6945.

5. Wang, S.; Cyronak, M.; Yang, E. Does a Stable Isotopically Labeled Internal Standard Always Correct Analyte Response? A Matrix Effect Study on a LC/MS/MS Method for the Determination of Carvedilol Enantiomers in Human Plasma. J. Pharm. Biomed. Anal. 2007, 43(2), 701-707

6. Wang, G.; Wu, W. W.; Zeng, W.; Chou, C. L.; Shen, R. F. Label-Free Protein Quantification Using LC-Coupled Ion Trap or FT Mass Spectrometry: Reproducibility, Linearity, and Application with Complex Proteomes. J. Proteome Res. 2006, 5(5), 1214-1223.

7. Silva, J. C.; Gorenstein, M. V.; Li, G. Z.; Vissers, J. P.; Geromanos, S. J. Absolute Quantification of Proteins by LCMSE: A Virtue of Parallel MS Acquisition. Mol. Cell. Proteom. 2006, 5(1), 144-156.

8. Weinmann, W.; Wiedemann, A.; Eppinger, B.; Renz, M.; Svoboda, M. Screening for drugs in serum by electrospray ionization/collisioninduced dissociation and library searching. J. Am. Soc. Mass Spectrom. 1999, 10(10), 1028-1037.

9. Bristow, A. W.; Nichols, W. F.; Webb, K. S.; Conway, B. Evaluation of Protocols for Reproducible Electrospray In-Source Collisionally Induced Dissociation on Various Liquid Chromatography/Mass Spectrometry Instruments and the Development of Spectral Libraries. Rapid Commun. Mass Spectrom. 2002, 16(24), 2374-2386.

10. Bogusz, M. J.; Maier, R. D.; Kruger, K. D.; Webb, K. S.; Romeril, J.; Miller, M. L. Poor Reproducibility of In-Source Collisional Atmospheric Pressure Ionization Mass Spectra of Toxicologically Relevant Drugs. J. Chromatogr. A 1999, 844(1/2), 409-418.

11. Gika, H. G.; Theodoridis, G. A.; Wingate, J. E.; Wilson, I. D. Within-Day Reproducibility of an HPLC-MS-Based Method for Metabonomic Analysis: Application to Human Urine. J. Proteome Res. 2007, 6(8), 3291-3303.

12. Prakash, A.; Piening, B.; Whiteaker, J.; Zhang, H.; Shaffer, S. A.; Martin, D.; Hohmann, L.; Cooke, K.; Olson, J. M.; Hansen, S.; Flory, M. R.; Lee, H.; Watts, J.; Goodlett, D. R.; Aebersold, R.; Paulovich, A.; Schwikowski, B. Assessing Bias in Experiment Design for Large Scale Mass Spectrometry-Based Quantitative Proteomics. Mol. Cell. Proteom. 2007, 6(10), 1741-1748.

13. Muller, C.; Schafer, P.; Stortzel, M.; Vogt, S.; Weinmann, W. Ion Suppression Effects in Liquid Chromatography-Electrospray-Ionization Transport-Region Collision Induced Dissociation Mass Spectrometry with Different Serum Extraction Methods for Systematic Toxicological Analysis with Mass Spectra Libraries. J. Chromatogr. B Analyt. Technol. Biomed. Life Sci. 2002, 773(1), 47-52.

14. Chambers, E.; Wagrowski-Diehl, D. M.; Lu, Z.; Mazzeo, J. R. Systematic and Comprehensive Strategy for Reducing Matrix Effects in LC/
MS/MS Analyses. J. Chromatogr. B Analyt. Technol. Biomed. Life Sci. 2007, 852(1/2), 22-34.

15. Bottcher, C.; Roepenack-Lahaye, E. V.; Willscher, E.; Scheel, D.; Clemens, S. Evaluation of Matrix Effects in Metabolite Profiling Based on Capillary Liquid Chromatography Electrospray Ionization Quadrupole Time-of-Flight Mass Spectrometry. Anal. Chem. 2007, 79(4), 1507-1513.

16. Annesley, T. M. Methanol-Associated Matrix Effects in Electrospray Ionization Tandem Mass Spectrometry. Clin. Chem. 2007, 53(10), 18271834.

17. Hopley, C.; Bristow, T.; Lubben, A.; Simpson, A.; Bull, E.; Klagkou, K.; Herniman, J.; Langley, J. Towards a Universal Product Ion Mass Spectral Library-Reproducibility of Product Ion Spectra Across Eleven Different Mass Spectrometers. Rapid Commun. Mass Spectrom. 2008, 22(12), 1779-1786.

18. Jansen, R.; Lachatre, G.; Marquet, P. LC-MS/MS Systematic Toxicological Analysis: Comparison of MS/MS Spectra Obtained with Different Instruments and Settings. Clin. Biochem. 2005, 38(4), 362-372.

19. Pavlic, M.; Libiseller, K.; Oberacher, H. Combined Use of ESIQqTOF-MS and ESI-QqTOF-MS/MS with Mass-Spectral Library Search for Qualitative Analysis of Drugs. Anal. Bioanal. Chem. 2006, 386(1), $69-82$.

20. Volna, K.; Holcapeck, M.; Kolarova, L.; Lemr, K.; Caslavsky, J.; Kacer, P.; Poutska, J.; Hubalek, M. Comparison of Negative Ion Electrospray Mass Spectra Measured by Seven Tandem Mass Analyzers Towards Library Formation. Rapid Commun. Mass Spectrom. 2008, 22(2), 101-108.

21. Mohan, K. R.; Bartlett, M. G.; Busch, K. L.; Scheoen, A. E.; Gore, N. Calibration Point for Electron Ionization MS/MS Spectra Measured with Multiquadrupole Mass Spectrometers. J. Am. Soc. Mass Spectrom. 1994, 5(6), 576-582

22. Lemire, S. W.; Busch, K. L. Calibration Point for Liquid Secondary Ion Mass Spectrometry Tandem Mass Spectra Measured with an EB Hybrid Mass Spectrometer. J. Mass Spectrom. 1996, 31(3), 280-288.

23. Olson, M. T.; Blank, P. S.; Sackett, D. L.; Yergey, A. L. Evaluating Reproducibility and Similarity of Mass and Intensity Data in Complex Spectra Applications to Tubulin. J. Am. Soc. Mass Spectrom. 2008, 19(3) 367-374.

24. Collette, C.; De Pauw, E. Calibration of the Internal Energy Distribution of Ions Produced by Electrospray. Rapid Commun. Mass Spectrom. 1998, 12, 165-170.

25. Derwa, F.; De Pauw, E.; Natalis, P. New Basis for a Method for the Estimation of Secondary Ion Internal Energy Distribution in 'Soft' Ionization Techniques. Organic Mass Spectrom. 1991, 26, 117-118.

26. Drahos, L.; Heeren, R. M.; Collette, C.; De Pauw, E.; Vekey, K. Thermal Energy Distribution Observed in Electrospray Ionization. J. Mass Spectrom. 1999, 34(12), 1373-1379.

27. Greisch, J. F., Gabelica, V.; Remacle, F.; De Pauw, E. Thermometer Ions for Matrix-Enhanced Laser Desorption/Ionization Internal Energy Calibration. Rapid Commun. Mass Spectrom. 2003, 17(16), 1847-1854.

28. Gabelica, V.; De Pauw, E. Internal Energy and Fragmentation of Ions Produced in Electrospray Sources. Mass Spectrom. Rev. 2005, 24(4), 566-587.

29. Nefliu, M.; Smith, J. N.; Venter, A.; Cooks, R. G. Internal Energy Distributions in Desorption Electrospray Ionization (DESI). J. Am. Soc. Mass Spectrom. 2007, 19(3), 420-427.

30. Gabelica, V.; Schulz, E.; Karas, M. Internal Energy Build-Up in MatrixAssisted Laser Desorption/Ionization. J. Mass Spectrom. 2004, 39(6), 579-593.

31. Busman, M.; Rockwood, A. L.; Smith, R. D. Activation Energies for Gas-Phase Dissociations of Multiply Charged Ions from Electrospray Ionization Mass Spectrometry. J. Phys. Chem. 1992, 96(6), 2397-2400.

32. Rockwood, A. L.; Busman, M.; Udseth, H. R.; Smith, R. D. Thermally Induced Dissociation of Ions from Electrospray Mass Spectrometry. Rapid Commun. Mass Spectrom. 1991, 5, 582-585.

33. Meot-Ner (Mautner), M.; Dongré, A. R.; Somogyi, A.; Wysocki, V. H. Thermal Decomposition Kinetics of Protonated Peptides and Peptide Dimers, and Comparison with Surface-Induced Dissociation. Rapid Commun. Mass Spectrom. 1995, 9(9), 829-836.

34. Gabelica, V.; De Pauw, E.; Karas, M. Influence of the Capillary Temperature and the Source Pressure on the Internal Energy Distribution of Electrosprayed Ions. Int. J. Mass Spectrom. 2004, 231, 189-195.

35. Nemes, P.; Marginean, I.; Vertes, A. Spraying Mode Effect on Droplet Formation and Ion Chemistry in Electrosprays. Anal. Chem. 2007, 79(8), 3105-3116.

36. Pak, A.; Lesage, D.; Gimbert, Y.; Vekey, K.; Tabet, J. C. Internal Energy Distribution of Peptides in Electrospray Ionization: ESI and CollisionInduced Dissociation Spectra Calculation. J. Mass Spectrom. 2007, 43(4), 447-455.

37. Daikoku, S.; Ako, T.; Kato, R.; Ohtsuka, I.; Kanie, O. Discrimination of 16 Structural Isomers of Fucosyl Galactoside Based on Energy-Resolved Mass Spectrometry. J. Am. Soc. Mass Spectrom. 2007, 18(10), 1873-1879.

38. Dole, M.; Mack, L. L.; Hines, R. L.; Mobley, R. C.; Ferguson, L. D.; Alice, M. B. Molecular Beams of Macroions. Chem. Phys. 1968, 49, 2240-2249.

39. Iribarne, J. V.; Thomson, B. A. On the Evaporation of Small Ions from Charged Droplets. J. Chem. Phys. 1976, 64(6), 2287-2294.

40. Cole, R. B. Some Tenets Pertaining to Electrospray Ionization Mass Spectrometry. J. Mass Spectrom. 2000, 35(7), 763-772.

41. Nguyen, S.; Fenn, J. B. Gas-Phase Ions of Solute Species from Charged Droplets of Solutions. Proc. Natl. Acad. Sci. U.S.A. 2007, 104(4), 11111117 . 
42. Touboul, D.; Jecklin, M. C.; Zenobi, R. Ion Internal Energy Distributions Validate the Charge Residue Model for Small Molecule Ion Formation by Spray Methods. Rapid Commun. Mass Spectrom. 2008, 22(7), 10621068.

43. Gabelica, V.; Lemaire, D.; Laprévote, O.; De Pauw, E. Kinetics of Solvent Addition on Electrosprayed Ions in an Electrospray Source and in a Quadrupole Ion Trap. Int. J. Mass Spectrom. 2001, 210/211, 113-119.

44. Kenttämaa, H. I.; Cooks, R. G. Internal Energy Distributions Acquired Through Collisional Activation at Low and High Energies. Int. J. Mass Spectrom. Ion Processes 1985, 64(1), 79-83.

45. Naban-Maillet, J.; Lesage, D.; Bossee, A.; Gimbert, Y.; Sztaray, J.; Vekey, K.; Tabet, J. C. Internal Energy Distribution in Electrospray Ionization. J. Mass Spectrom 2005, 40(1), 1-8.

46. Krutchinsky, A. N.; Chernushevich, I. V.; Spicer, V. L.; Ens, W.; Standing, K. G. Collisional Damping Interface for an Electrospray Ionization Time-of-Flight Mass Spectrometer. J. Am. Soc. Mass Spectrom. 1998, 9, 569-579.

47. Hang, W.; Lewis, C.; Majidi, V. Practical Considerations When Using Radio Frequency-Only Quadrupole Ion Guide for Atmospheric Pressure Ionization Sources with Time-of-Flight Mass Spectrometry. Analyst 2003, 128, 273-280.
48. Haas, K.; Feldmann, J.; Wennrich, R.; Stark, H.J. Species-Specific Isotope-Ratio Measurements of Volatile Tin and Antimony Compounds Using Capillary GC-ICP-Time-of-Flight MS. Fresenius J. Anal. Chem. 2001, 370(5), 587-596.

49. Zhang, H.; Heinig, K.; Henion, J. Atmospheric Pressure Ionization Time-of-Flight Mass Spectrometry Coupled with Fast Liquid Chromatography for Quantitation and Accurate Mass Measurement of Five Pharmaceutical Drugs in Human Plasma. J. Mass Spectrom. 2000, 35(3), 423-431.

50. Lecchi, P.; Zhao, J.; Wiggins, W.; Bertenshaw, G.; Chen, T.; Mansfield, B.; Ping, Y.; Peltier, J. M. Defining Instrument Performance and Assessing the Reproducibility of Mass Spectrometric Analyses of Complex Samples. Proceedings of the 56th ASMS Conference on Mass Spectrometry and Allied Topics; 2008 June, Denver, CO.

51. De Maesschalck, R.; Jouan-Rimbaud, D.; Massart, D. L. The Mahalanobis Distance. Chemomet. Intel. Lab. Syst. 2000, 50(1), 1-18.

52. Press, W. H.; Teukolsky, S. A.; Vetterling, W. T.; Flannery, B. P. In Numerical Recipes: The Art of Scientific Computing, 3rd ed., Cowles, L.; Harvey, A. Eds.; Cambridge University Press: New York, 2007; p. 1256. 